\title{
Fournier's Gangrene: Descriptive Analysis and Outcome of Eleven Cases
}

\author{
J arboui $\mathrm{S}^{1 *}$, Bekkey MA' ${ }^{2}$, Triki $\mathrm{H}^{1}$, Farhat $\mathrm{I}^{1}$ and \\ Lepandatu $\mathrm{A}^{2}$ \\ ${ }^{1}$ Department of General Surgery, Mahmoud Matri \\ Hospital, Ariana, Tunisia \\ ${ }^{2}$ Department of General Surgery, Sidi Bouzid Hospital, \\ Tunisia \\ *Corresponding author: Slim J arboui, Department \\ of General Surgery in Mahmoud Matri Hospital, Ariana, \\ Tunisia
}

Received: December 05, 2016; Accepted: February 08, 2017; Published: February 10, 2017

\author{
Abstract \\ Background: Fournier's Gangrene (FG) is a rare but potentially life \\ threatening extensive fasciitis of the external genitalia and perineum.
}

Aim of the Study: The aim is to describe the clinical features of patients treated on for FG and their hospital outcome after surgery.

Patients and Methods: Between 2010 and 2014, 11 patients with FG were enrolled in the department of general surgery of Sidi Bouzid Hospital - Tunisia.

Results: Eight male and three women were enrolled in the study. Six patients had diabetes. All patients were treated with common approach board spectrum antibodies, wide surgical excision with systematic revision. Three patients had had Hyperbaric Oxygen Therapy (HOT) and three had had colostomy. One patient treated with vacuum therapy. The course was uneventful in 10 cases and one patient died of pulmonary embolism.

Conclusion: Early diagnosis and management are essential in the case of FG because the risk of rapid extension and overwhelming sepsis is real leading to high rate of mortality.

Keywords: Fournier's gangrene; Sepsis; Perineal gangrene; Surgical excision

\section{Introduction}

Fournier's Gangrene (FG) is a life-threatening necrotizing fasciitis of the perineal, genital and perineal regions. Mortality has been reported in different series to range from 20 to $50 \%$ [1-2]. This disease is worldwide and, is recognized more frequently among male adults. Early surgical debridement and administration of broadspectrum antibiotics are fundamental in the treatment of FG. Surgical reconstruction may follow when necessary.

The purpose of this study is to report our experience over the past 5 years regarding the management of FG and to assess retrospectively the rate of the severity and its reliability to predict the post operative outcome.

\section{Patients and Methods}

The medical records of all consecutives patients with Fournier's Gangrene (FG) who were treated in the department of General Surgery at Sidi Bouzid Hospital -Tunisia between 2010 and 2014. Data were retrospectively collected about medical history and co morbidities, symptoms, physical examination, laboratory tests, imaging results, etiology, timing of surgical excision, antibiotics treatment, extent of surgical debridement, length of stay in the Unit of Intensive Care (ICU), respiratory assistance, number of excision under anesthesia, length of hospital stay and the post treatment course (mortality).

Diagnosis of FG was made on clinical characteristics in all patients with local sign of edema, erythema, tenderness, bleb formation or gangrene on physical examination.

The topography extent of the fasciitis and necrosis was appreciated pre and per operatively (confirmed by photos) ranging from those localized in the perineal region, those extended to the fesses region and/or to the roots of the lumbs, and/or to the abdominal or chest wall.

We calculated retrospectively the Fournier's Gangrene Severity Index Score (FGSIS) for all patients to evaluate the gravity of the pathology. In this score, nine parameters were calculated: temperature, heart rate, respiratory rate, serum sodium, potassium, creatinine, bicarbonates level, hematocrit and leukocyte count.

Cephalosporin third generation, aminoglycosides and Metronidazole were used as combination in all patients. Surgical debridement was performed in emergency with extensive and aggressive approach depending on the extent of the necrotic tissues. Culture samples were taken from the wounds or pus and addressed for bacteriologic analysis. Aggressive debridement with resection of the necrotic skin, subcutaneous tissue, fascia and muscle until viable tissue was reached. Systematic revision under anesthesia was performed for all patients 24 to 48 hours after the first surgery. The bedside debridement was usually performed under general anesthesia. The number of iterative surgical revision, dressing change in the operating room and the indication of colostomy depends on the local evolution and the risk of fecal contamination of the operative wound.

\section{Results}

Between 2010 and 2014, eleven patients with FG were enrolled in the department of General Surgery at the Sidi Bouzid Hospital in Tunisia. The average age was 38.2 years (range 17-65 years). There were eight male and three women. Six patients had diabetes mellitus 


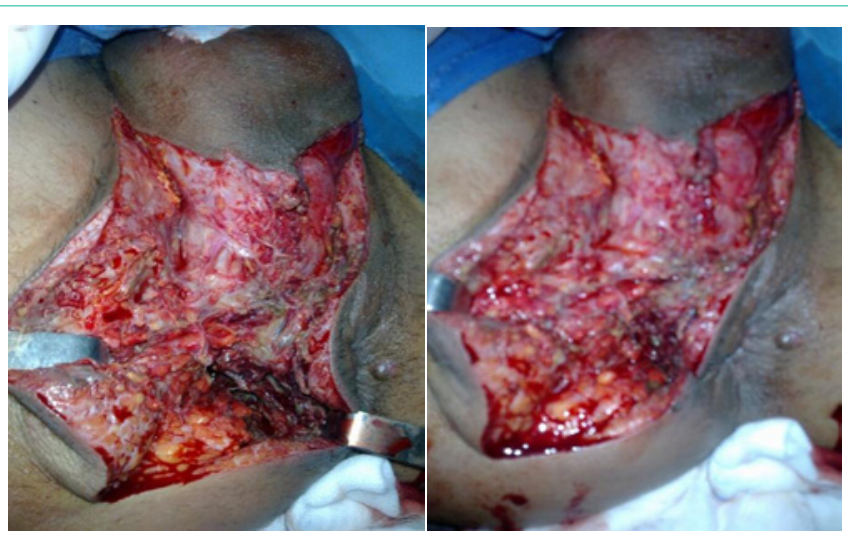

Figure 1: Fournier's gangrene complicating perineal abscess in a diabetic patient (Obs.3).
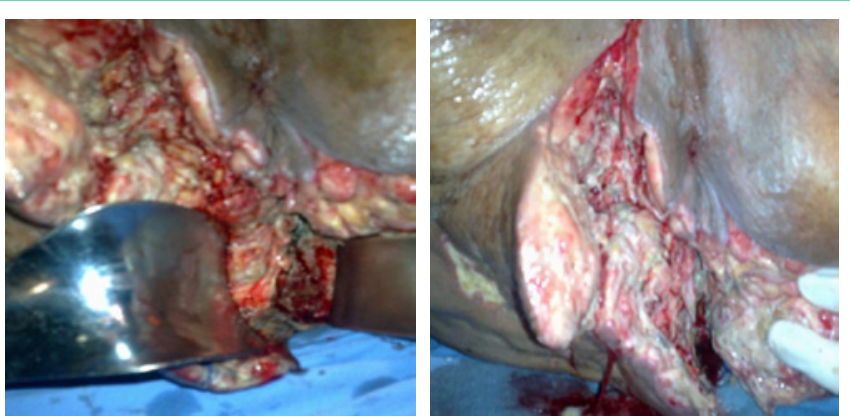

Figure 2: Fournier's gangrene complicating ischio rectal abscess (Obs.2)

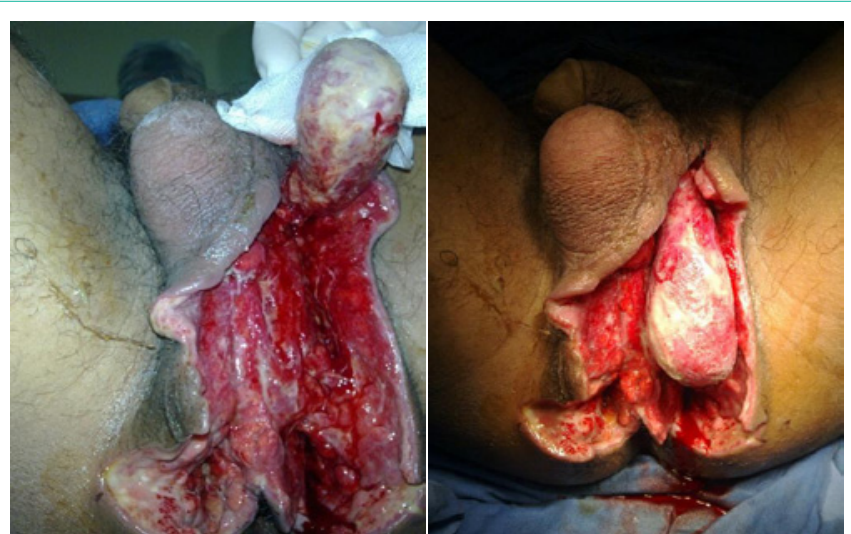

Figure 3A: Fournier's gangrene in a man after hemoroidectomy. View after first surgical debridement (Obs.4).

and two were obese. The most common etiologic factor for FG was perineal abscess (six cases). Figure 1 and Figure 2 describe the local examination of two patients of them (Obs. 2 and 3). In one case, FG had complicated the post operative course of hemorrhoidectomy (Obs.4 and Figure 3A) and in one case (Obs. 5), the etiology was a bartholinite (Figure 4). The mean time to diagnosis was 4.3 days (range: 1-8 days).

In all cases, the diagnosis was made by clinical examination. Among symptoms at admission, perineal pain was found in all patients and pyrexia was present in ten patients. One patient had initial shock (Obs. 9). The extent of the necrotizing process was

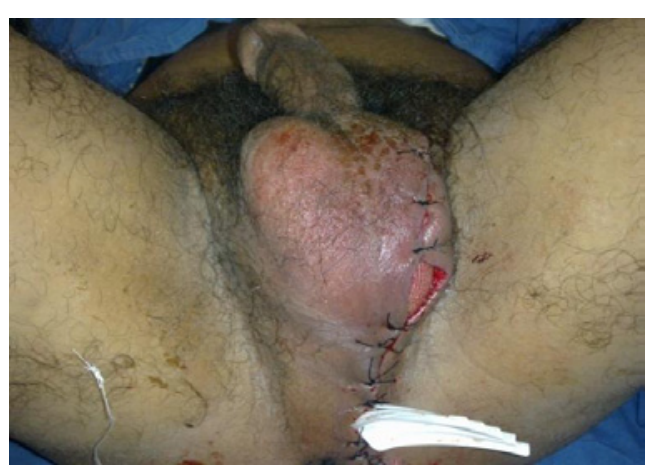

Figure 3B: The outcome of the surgical site after three complementary revisions under anesthesia.

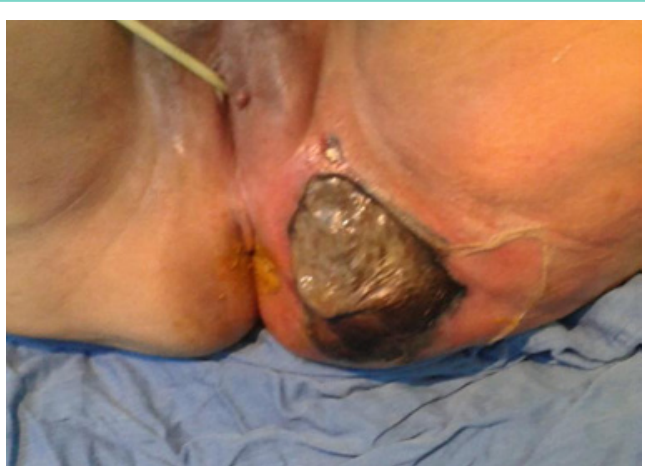

Figure 4: Fournier's gangrene complicating bartholinite (Obs.5).

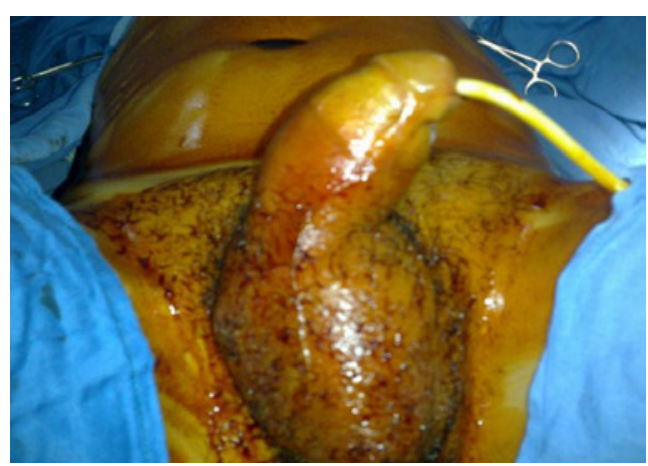

Figure 5A: Operative view: edema and swelling of the scrotum and the anorectal region (Obs.1).

assessed by the physical examination and by Computed Tomography (CT) in ten cases. In seven cases, the cellulitis was limited to the perineum, in two cases, it was extended to the roots of the limbs (Obs. 3 and 8), in two cases to the abdominal wall (Obs. 2 and 4) and in one situation to the chest wall (Obs. 1). In six cases, there was no myonecrosis, but in five cases, myonecrosis was localized or extended to the gluteal muscle (Obs. 5) or to the adductor and / or flanks (Obs. 1; 3; 4 and 8). Three patients had been presented in the emergency with perineal gas gangrene complicating in all cases perineal abscess. Bacterial gas production was revealed by subcutaneous crackling. Figure $5(\mathrm{~A}+\mathrm{B})$ illustrated the clinical aspect and the CT result of gas gangrene diagnosed in a 17- year - old men (Obs. 1). The mean of the Fournier's Gangrene Severity Index Score (FGSIS) was 4.18 (range: 1-9). The characteristics data of each patient of our series were 


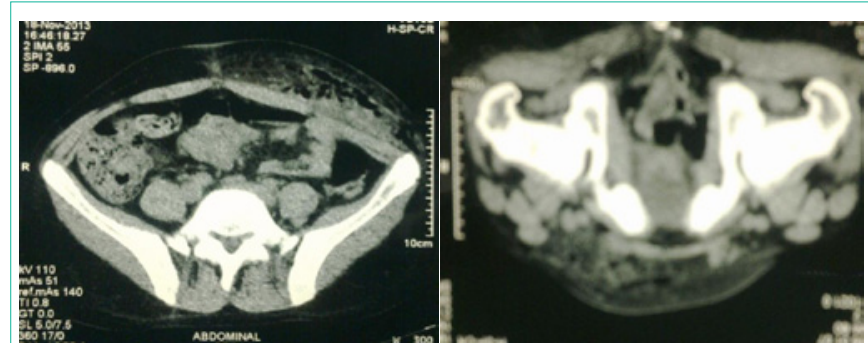

Figure 5B: Computed tomography showing the extent of the necrotic process in the pelvis (pelvirectal abscess) with gas infiltration into the anterior abdominal wall.

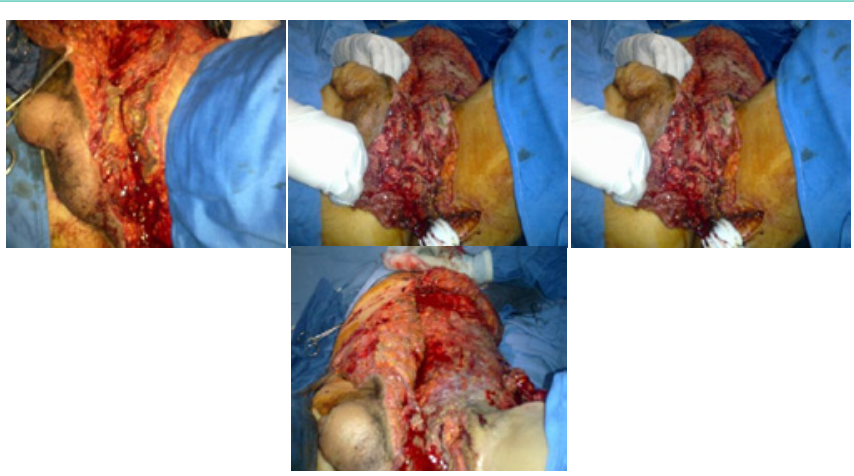

Figure 5C: Operative view after extensive and aggressive surgical debridement of all died skin and subcutaneous tissue involved.

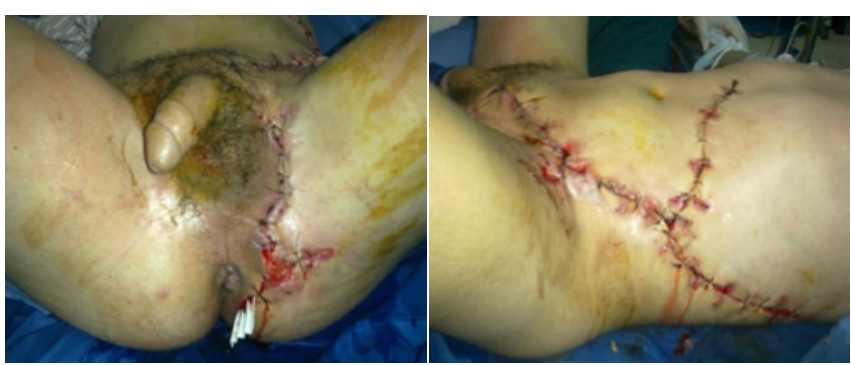

Figure 5D: The local evolution after surgical debridement and iterative revision of the bedside (14 times) leading to secondary reconstruction.

collected in Table1.

All patients had an almost standardized treatment consisted on antibiotic therapy, resuscitation and surgery. Resuscitation consisted of volume expansion, correction of metabolic and electrolytic disorders if presents. All patients had transurethral catheterization. Antibiotic therapy was introduced since admission. It was a probabilistic antibiotherapy covering anaerobic bacteria, gram-negative bacilli and gram-cocci-positive. The combination cephalosporin third generation-gentamicin and metronidazole was prescribed in all patients. Cultures of pus and tissue sample were performed in eight patients. Bacteriologic studies were positive in four cases and revealed Escherichia coli in three patients (Obs. 2,4,8) and C. Perfringens in Obs. 1. Surgery was performed under general anesthesia and comprised wide cutaneous, subcutaneous and muscle excision up to healthy and viable tissue. Large drainage sets were used on the subcutaneous and muscle detachments. Figure 5C illustrated the extent of surgical excision and debridement in a patient admitted for perineum gas gangrene. All patients had iterative surgical revision initially under general anesthesia and then without anesthesia. The number of revision ranged from 3 to 12 with a mean of 6.18 . Three patients had lateral colostomy (Obs. 2; 7 and 8). Three patients had had hyperbaric oxygen therapy (Obs. 2; 4 and 10). The procedure was started ten to 15 days after surgery. They were exposed to pure oxygen at 2.5 atmospheres absolute pressure for 100 to $120 \mathrm{~min}$. this procedure was repeated three times weekly for respectively one, two and two months. One patient had a negative therapy procedure by vacuum as adjunct therapy to surgery (Obs. 11). Five patients had respiratory assistance for 24 to 48 hours in the intensive care unit because of the gravity of sepsis (Shock in Obs. 9) or relative instability of the hemodynamic status after extensive surgery. Five patients had had sanguine transfusion. The length of the hospital stay ranged from 13 to 54 days with a mean of 29 days. The post operative course was uneventful in all but one patient whose dead from pulmonary embolism despite good local evolution (Obs. 9). The Table 2 summarized the management and the course of patient's series.

No patients from our series had a plastic reconstructive procedure. In all of them, the wounds were followed up for secondary healing. Figure 3A and Figure 5D showed the good local evolution after iterative revision and secondary approximation suture (Obs. 1 and Obs. 4). For patients who had colostomy, restorative continuity was done four months after the initial surgery.

\section{Discussion}

Fournier's Gangrene was originally described as scrotal gangrene in young males. Actually, it is accepted as synergetic necrotizing fasciitis of perineal, genital, and perineal region. This disease can occur at any age but predominates in young males between 40 and 50 years [1-5]. In our series, the mean age was 38.2 years with male predominance (8/3). It has been shown that FG affects patients with obvious immune compromise such as diabetes, alcoholism, immunosuppression, neoplasic disease and obesity. In the series of çakmak et al, $44.6 \%$ of patients had diabetes [6], 20\% in the review of Eke et al compiling 1726 cases [1], and in our previous published series with 35 patients, 23 had diabetes [7]. Regarding etiologies, the anorectal origin seemed to be the more frequent one: $37 \%$ in the series of Brunet et al [5], $42 \%$ in the work of EL Mejjed et al [8], 42\% I the series of Yilmazlar et al compiling 120 patients from one center [9], $72 \%$ in our series. Anorectal origins include fistulas, fissures, abscess of anal margins. We didn't note patients with FG secondary to urogenital causes because they were supported in the urology department.

Diagnosis of FG is based on clinical symptom and examination: scrotal and/or perineal edema, parietal necrosis, indurations, crepitations, fever. Systemic symptoms may include leukocytosis, dehydratation, tachycardia, anemia and hyperglycemia. The principal role of imaging and particularly computed tomography is to permit an accurate assessment of the disease extent. Findings at $\mathrm{Ct}$ include asymmetric fascial thikening, collections and abscess, subcutaneous emphysema. It helps also to assess the retroperitoneum to which the disease can spread and could explain the cause of non improvement after surgery [7,10-12]. In our series, 10 patients had CT imaging before surgery that was very helpful to assess the extent of the disease without delaying surgery. 
Table 1: Patients characteristics.

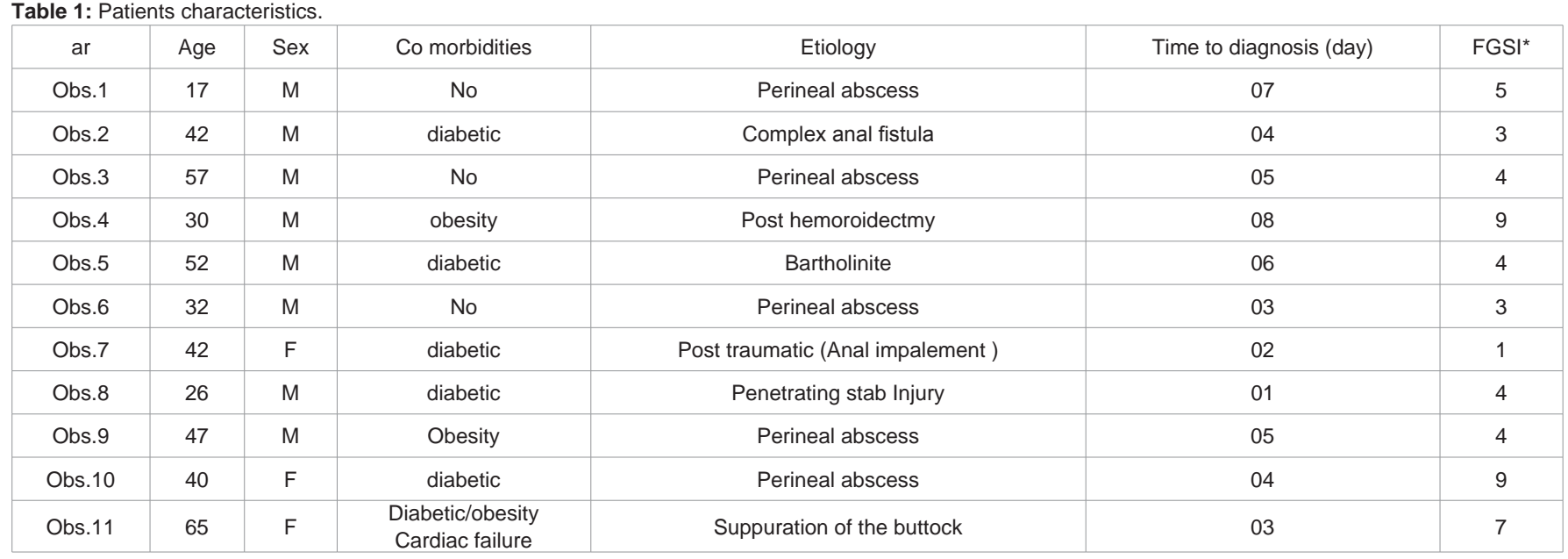

Fournier's Gangrene Sepsis Index Severity.

Table 2: Patient's management and hospital outcome.

\begin{tabular}{|c|c|c|c|c|c|c|}
\hline Patients & Number of surgical revision & Colostomy & Respiratory assistance & $\begin{array}{l}\text { ICU } \\
\text { (day) }\end{array}$ & $\begin{array}{l}\text { Stay hospitalization } \\
\text { (day) }\end{array}$ & Post operative course \\
\hline Obs.1 & 12 & No & No & 02 & 32 & Uneventful \\
\hline Obs.2 & 06 & Yes & yes & 04 & 28 & Uneventful \\
\hline Obs.3 & 08 & No & No & 02 & 30 & Uneventful \\
\hline Obs. 4 & 03 & No & No & 0 & 34 & Uneventful \\
\hline Obs.5 & 08 & No & Yes & 02 & 18 & Uneventful \\
\hline Obs.6 & 04 & No & No & 0 & 30 & Uneventful \\
\hline Obs.7 & 03 & yes & Yes & 02 & 23 & Uneventful \\
\hline Obs.8 & 05 & yes & No & 0 & 16 & Uneventful \\
\hline Obs.9 & 06 & No & Yes & 2 & 54 & Dead \\
\hline Obs.10 & 05 & No & No & 0 & 13 & Uneventful \\
\hline
\end{tabular}

FG tends to be a polymicrobial disease, with synergy of aerobic and anaerobic bacteria. The most commonly bacteria found are Escherchia Coli, Bacteriodes, streptococcal species, Klebsiella, clostridium and pseudomonas. [1,2,5,7]. . Cultures of pus and tissue sample were performed in eight patients. Bacteriologic studies were positive in four cases and revealed Escherichia coli in three patients (Obs. 2,4,8) and C. Perfringens in Obs. 1. Three of our patients were admitted for gas gangrene with evident crepitus at examination. This clostridial-myonecrosis is characterized by the presence of gas under the skin which is produced by clostridium. Tissue necrosis is due to the production of endotoxins by spore gas producing bacteria.

Perineal gangrene is a therapeutic emergency. The management includes surgical debridement, antibiotic therapy and intensive care. Intensive care consists of restoration of fluid and electrolytes balance. Initial antimicrobial treatment must cover aerobic and anaerobic bacteria. Ampicillin-Sulbactam or piperacillin-Tazobactam or Ticarcillin-Claculateare is suggested empiric regimens. It is secondarily adapted to the antibiogram $[1,2,5,7,13,14]$. The more prescribed combination in our series was Cephalosporin third generation-Aminoglycoside and Metronidazole.

Surgery consists of debridement and excision of necrotic tissue up to healthy margins. We advocate, as for most authors, a highly aggressive debridement since the initial operation, because leaving any focus of infection could act as a starting point to sepsis $[1,2,7]$. In our policy, we do systemically a second look operation $24-48$ hours after the initial one, to ensure that all necrotic tissue has been cleared and as advocated by Brunet's team and others [13]. Iterative excision in the operating room is often necessary [14-16]. During treatment, testicles are usually spared because they have a separate blood supply originating from the abdominal aorta.

Foley's urethral catheter was performed in all of our patients, and is usually advocated for urinary diversion. Colostomy is mandatory for some authors $[5,13]$ and optional for others $[2,6,13,14]$. It avoids fecal contamination of the wound and facilitates local healing. In our opinion, because it increases morbidity, colostomy should be reserved only for selected patients with lesions involving the anorectal area and sphincter with a high risk of fecal contamination of the bedside. In our series, only three patients had colostomy. Restorative continuity should be performed once the wound heals and after making sure about the continence of the anal sphincter.

Adjunctive HBOT has been shown to increase wound healing, decrease the gangrenous spread in conjunction to surgery and antibodies and so reduces the number of debridement required. HBOT reduces the hypoxic dysfunction of leucocytes, has a direct 
antibacterial effect against anaerobic bacteria and plays a role in wound healing and angiogenesis $[4,7,17,18]$. Three of our patients were treated by HBOT as adjunct therapy to surgery.

Some authors have shown the vacuum therapy is effective in the management of large wounds and that decrease significantly the length of hospital stay, offers fewer dressing changes and less pain $[2,4]$. One patient in our series had been treated with this procedure of negative therapy and dressing with good local course.

The surgical wounds are usually left for secondary healing or delay primary wound closure. In some cases, after healthy granulation has appeared, and where surgery excision left a large tissue defect, reconstructive procedure with local skin flaps or grafts could be indicated $[4,7,8,13,20]$. In the systematic review of Karian et al, about the reconstruction defect after FG, they conclude that skin grafting or flap reconstruction is recommended for defects larger than $50 \%$ of the scrotum or extended beyond the scrotum. For the defect less than $50 \%$ of the scrotum primary closure without tension could be indicated or scrotal advancement flap reconstruction or secondary intention [21].

Regarding prognosis, FG is a serious disease with a mortality rate varying from $0 \%$ to $88 \%[1,2,4,7]$. In the largest series of 1756 patients, Eke et al reported a mortality rate of $16 \%$ [1]. In the study of 1680 patients, Sorensen et al reported a mortality rate of $7.5 \%$ in men and $19.8 \%$ in women [2]. The principals causes of death in the case of FG are severe sepsis, coagulopathy, acute kidney failure, and multiple organ failure [4,7]. In some series, advanced age, therapeutic delay, extent of myonecrosis, FGSIS, positive culture for streptococcus have been identified as negative factors for prognosis [1,2,4,7,12,13,22 24]. The FGSIS is a numeric score proposed by Laor et al in 1995 to establish a prognosis of the outcome of the disease. It is obtained from a combination of admission physiologic parameters [22]. It has been proposed that a FGSIS > 9 indicates a $75 \%$ probability of mortality. In our series, the mean of FGSIS was 4.18 (only one patient had a score $>9$ ) and the mortality rate was $9 \%$. This rate of death could be explained by the fact that our patients were relatively young, the interval between the onset of the disease and diagnosis is short (mean: 4.3 days) and the low rate of FGSIS which enabled us to start the aggressive surgical treatment in time.

\section{Conclusion}

FG is a life threatening disease with high morbidity and mortality. Early diagnosis and instauration of treatment based on antibiotic therapy, intensive care and aggressive surgical debridement are the major prognostic factors. HBOT and vacuum therapy should be considered in the therapeutic arsenal.

\section{References}

1. Eke N. Fournier's gangrene: A review of 1726 cases. Br J Surg 2000; 87: 718-728.

2. Sorensen MD, Krieger JN, Rivara FP, Broghammer JA, Klein MB, Mack CD et al. Fournier's Gangrene: population based epidemiology and outcomes. J Urol 2009; 181: 2120-2126.

3. Sroczynski M, Sebastian M, Rudnicki J, Sebastian A, Agrawal AL. A complex approach to the treatment of Fournier's Gangrene. Adv Clin Rxp Med. 2013; 22: $131-135$
4. Sarkis P, Farran F, Khoury R, Kamel G, Nemr E, Biajini J, et al. Fournier's Gangrene: A review of the recent literature. Progrès en Urologie 2009; 19: 75-84.

5. Brunet $C$, Consentino B, Barthelemy $A$, Huart L. Gangrènes périnéales: nouvelles approches bactériologiques. Résultats du traitement médicochirurgical (81 cas). Ann Chir. 2000; 125: 420-427.

6. çakmak A, Genç V, Akyol C. Fournier's Gangrene: Is it Scrotal Gangrene? Adv Ther 2008; 25: 1065-1074.

7. Jarboui S, Jarraya H, Daldoul S, Sayari S, Moussa MB, Abdesselem MM. Perineal gangrene: clinical and therapeutic features and prognostic analysis of 35 cases. Presse Med. 2008; 37: 760-766.

8. El Mejjad A, Belmahi $A$, Choukri $A$, Kafih M. La gangrène périnéo-scrotale: à propos de 31 cas. Ann Urol 2002; 36: 277-285.

9. Yilmazlar T, Isik Ozgen, Ozturk E, Ozer A, Gulcu B, Ercan I. Foutnier's Gangrene: Review of 120 patients and predictors of moratlity. Ulus Trauma Acil Cerrahi Derg 2014; 20: 333-337.

10. Özhan Oktar S, Yücel C, Tokgöz Ercan N, Çapkan D, Özdemir H. Fournier's gangrene: US and MR imaging findings. Eur J Radiol. 2004; 50: 81-87.

11. Morrison D, Blaivas M, Lyon M. Emergency diagnosis of Fournier's gangrene with bedside ultrasound. Am J Emerg Med. 2005; 23: 544-547.

12. Levenson RB, Singh AK, Novelline RA. Fournier Gangrene: Role of imaging. Radiographics 2008; 28; 519-528.

13. Brunet C, Dlpero JR, Guerinel G, Geisser A, Barthélémy A, Sainty JM et al. Gangrènes du périnée. Plaidoyer pour une conduite thérapeutique standardisé à propos de 50 observations. Chirurgie. 1992; 118: 607-614

14. Tazi K, Karmouni T, El Fassi J, El khader K. Gangrène périnéo scrotale : à propos de 51 cas. Aspects diagnostiques et thérapeutiques. Ann Urol. 2001; 35: 229-233.

15. Cem Ozden Y, Tufan S, Murat A. Fournier's gangrene: experience with 25 patients and use of Fournier's Gangrene Severity Index Score. Urology. 2004; 64: 218-222

16. Chawla SN, Gallop C, Mydlo JH. Fournier's gangrene: An analysis of repeated surgical debridement. Eur Urol 2003; 43: 572-575.

17. Riseman JA. Hyperbaric oxygen therapy for necrotizing fasciitis reduces mortality and the need for debridements. Surgery. 1990; 154: 847-850

18. Korhonen K, Hirm M, Niinikoski J. Hyperbaric Oxygen in the treatment of Fournier's Gangrene. Eur J Surg. 1998; 164: 251-255.

19. Czymek R, Schmidt A, Eckmann C, Boucher R, Wulff B, Laubert T, et al. Fournier's Gangrene: Vacuum-Assisted Closure versus Conventional Dressing. The American Jornal of Surgery. 2009; 197: 168-176.

20. Verna G, Fava F, Baglioni E, Cannatà M, Devalle L, Fraccalvieri M. La gangrene de Fournier: remarques sur deux cas cliniques. Ann Chir Plast Esthet. 2003; 49: 37-42

21. Karian LS, Chung SY, Lee ES. Reconstruction of defects after Fournier Gangrene: A Systematic review. Eplasty. 2015; 18.

22. Laor E, Palmer LS, Tolia BM, Reid RE, Winter HI. Outcome prediction in patients with Fournier's gangrene. J Urol 1995; 154: 89-92.

23. Yeniyol CO, Suelozgen T, Arslan M, Ayder AR. Fournier's Gangrene: Experience with 25 patients and use of Fournier's gangrene severity index score. Urology 2004; 64: 218-224

24. Corcoran AT, Smaldone MC, Gibbons EP, Walsh TJ, Davies BJ. Validation of the Fournier's Gangrene Severity Index in a large contemporary Series. J Urol. 2000; 180: 944-948. 